\title{
Optically sensitive Medipix2 detector for adaptive optics wavefront sensing
}

\author{
John Vallerga $\mathrm{a}^{\mathrm{a}}$, Jason McPhate ${ }^{\mathrm{a}}$, Anton Tremsin ${ }^{\mathrm{a}}$, Oswald Siegmund ${ }^{\mathrm{a}}$, Bettina \\ Mikulec $^{\mathrm{b}}$ and Allan Clark \\ ${ }^{a}$ University of California, Berkeley, 7 Gauss Way, Berkeley, CA, 94720-7450 USA \\ ${ }^{b}$ University of Geneva, 24, quai Ernest-Ansermet, 1211 Geneva 4, Switzerland
}

\begin{abstract}
A new hybrid optical detector is described that has many of the attributes desired for the next generation adaptive optics (AO) wavefront sensors. The detector consists of a proximity focused microchannel plate (MCP) read out by multi-pixel application specific integrated circuit (ASIC) chips developed at CERN ("Medipix2") with individual pixels that amplify, discriminate and count input events. The detector has 256 x 256 pixels, zero readout noise (photon counting), can be read out at $1 \mathrm{kHz}$ frame rates and is abutable on 3 sides. The Medipix 2 readout chips can be electronically shuttered down to a temporal window of a few microseconds with an accuracy of 10 nanoseconds. When used in a Shack-Hartmann style wavefront sensor, a detector with 4 Medipix chips should be able to centroid approximately 5000 spots using $7 \times 7$ pixel sub-apertures resulting in very linear, offnull error correction terms. The quantum efficiency depends on the optical photocathode chosen for the bandpass of interest.
\end{abstract}

\section{Introduction}

Ground-based astronomy in the optical and infrared has the distinct disadvantage of observing through the atmosphere. Though mostly transparent over much of the bandpass, the atmosphere is a constantly changing and dynamic medium. The index of refraction of air is a function of density and temperature and the vertical spatial profile of these parameters change with time due to windshear induced turbulence. Hence, a plane wave of light from a distant star will be distorted across the constant phase wavefront, leading to blurry images in the focal plane of a telescope or twinkling stars to the human eye.

\footnotetext{
"Corresponding author. Tel.: 1-510-643-5666; fax: 1-510-643-9729; e-mail: jvv@ssl.berkeley.edu.
} 
Ideally, the angular resolution of a telescope would be limited only by the diffraction limit of the primary mirror, which improves linearly with diameter. The typical $\sim 1$ arc second blurring caused by the atmosphere corresponds to the diffraction limit of a $20 \mathrm{~cm}$ diameter mirror in the optical bandpass. Therefore, making a ground-based telescope larger than $20 \mathrm{~cm}$ does not improve its angular resolution (though it does improve its light gathering power). Space-based telescopes, like the Hubble Space Telescope, do not suffer from atmospheric distortion, but they are expensive to build, launch and operate, and therefore difficult to make much larger.

In the past two decades, techniques have been developed to remove the effects of the atmosphere on the light from distant sources. Adaptive optics is the method of using fast deformable mirrors [1] to conjugate and therefore cancel any phase errors introduced in the light path between the object of interest and its image at the focal plane (Fig. 1). Before correcting the phase of the light, it must be measured using a wavefront sensor that samples the wavefront across the pupil. One method (among many) is the Shack-Hartmann sensor [1] (Fig 2). The wavefront is sampled by a lenslet array creating individual images focused on an imaging array. If the light is perfectly collimated (e.g. a plane wave from a distant star), the images would be spots of light at the regular spacing of the lenslet array. If the plane wave is distorted, the centroids of the spots would spatially shift depending on the local slope of the wavefront. By measuring the centroids of these spots in real time, one can determine the wavefront error and feed this error signal back to the deformable mirror to "close the loop" and correct all time variable wavefront distortions.

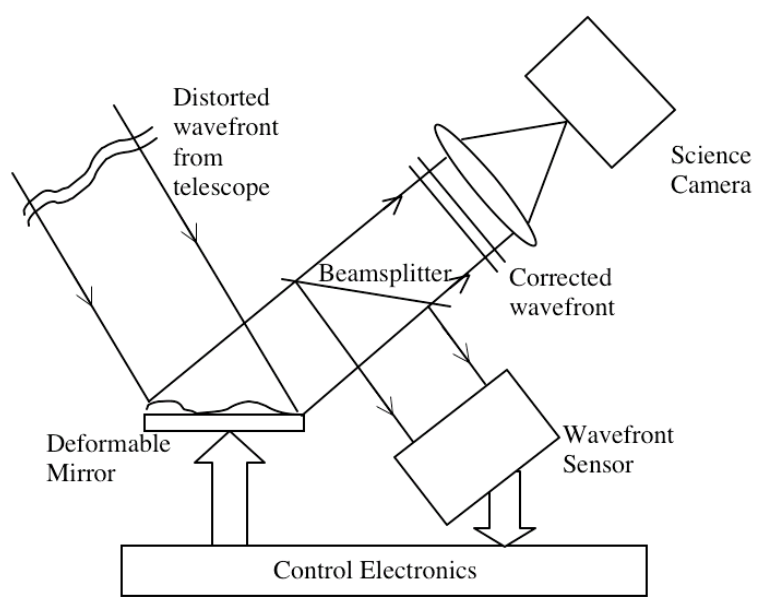

Figure 1. Simple schematic of an adaptive optics system where the aberrated wavefront is reflected off the deformable mirror resulting in a corrected diffraction limited wavefront passed on to a downstream camera. The beamsplitter takes a sample of the corrected wavefront to monitor the phase and send updates to the control electronics to "close the loop" to the deformable mirror.

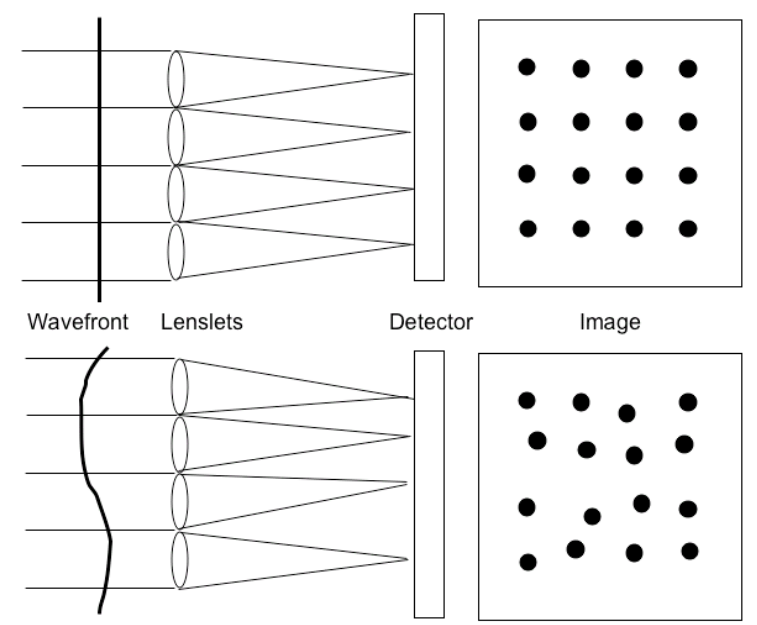

Figure 2. Cartoon to show how a Shack Hartmann wavefront sensor works. An input plane wave (top) is focused by a lenslet array resulting in a linear grid of stellar images on the $2 \mathrm{~d}$ detector. If the wavefront is aberrated (bottom), the centroids of the stellar image move away from the original grid. The amount of movement is a function of the slope of the wavefront; hence a Shack-Hartmann wavefront sensor measures the first derivative of the phase of the wavefront.

Each centroid determination measures the slope of the constant phase wavefront at that particular location on the wavefront at that particular time. Larger telescopes with larger pupils use deformable mirrors with more actuators and hence more phase 
measurements, therefore requiring detectors with many pixels. The atmosphere's variability on most nights necessitates wavefront sampling rates on the order of 100 to $1000 \mathrm{~Hz}$. The number of photons from the guide stars used as the reference beacon are almost always limited in number since bright stars are rare, so the wavefront sensor detector should have high quantum efficiency and low readout noise to improve the signal to noise ratio of the centroid determinations.

A recent white paper on the $\mathrm{AO}$ instrumentation needs for future large ( $>30 \mathrm{~m}$ diameter) telescopes entitled "A Roadmap for the Development of Astronomical Adaptive Optics" [2] specified that wavefront sensor detectors should have:

- Quantum efficiencies $>80 \%$

- Pixel formats of 512 x 512

- Frame rates of $1 \mathrm{kHz}$ or faster

- Readout noise less than 3 electrons rms.

The last three are not simultaneously achievable with the current generation of CCDs. Larger detectors require faster clocking rates to read out at fast frame rates. But higher clocking speeds increase the readout noise because of the increase in bandwidth required of the amplifiers. Below we describe a detector that can meet the last three of the above requirements, but with a quantum efficiency approaching $40 \%$.

\section{Photon counting MCP detectors}

Photon counting detectors, unlike charge integrating arrays like CCDs, register each detected photon as one count and so have no "readout noise". A variety of readout anodes are used in imaging microchannel plate (MCP) detectors (Fig. 3) to report the location and time of arrival of every detected photon. Imaging MCP detectors can have large area $(100 \times 100 \mathrm{~mm})$, high spatial resolution $(20 \mu \mathrm{m}$ FWHM), low background dark count, and event timing resolution less than 1 nanosecond [3]. Their $\mathrm{QE}$ is determined by the characteristics of the photocathode material that absorbs the initial photon and releases the photoelectron. The optical QE of these photocathodes is now approaching 50\%, in particular gallium arsenide (GaAs) (Fig. 4).

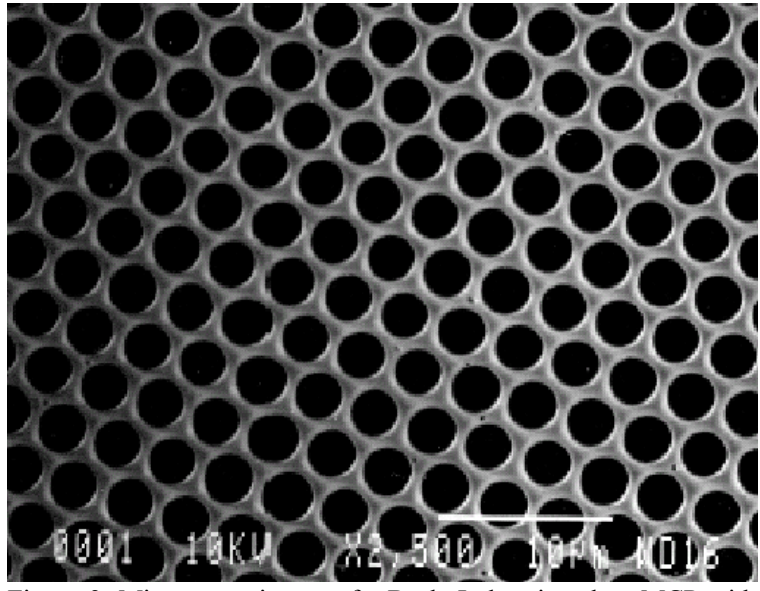

Figure 3. Microscope image of a Burle Industries glass MCP with $2 \mu \mathrm{m}$ pore diameters and $3 \mu \mathrm{m}$ pore spacing.

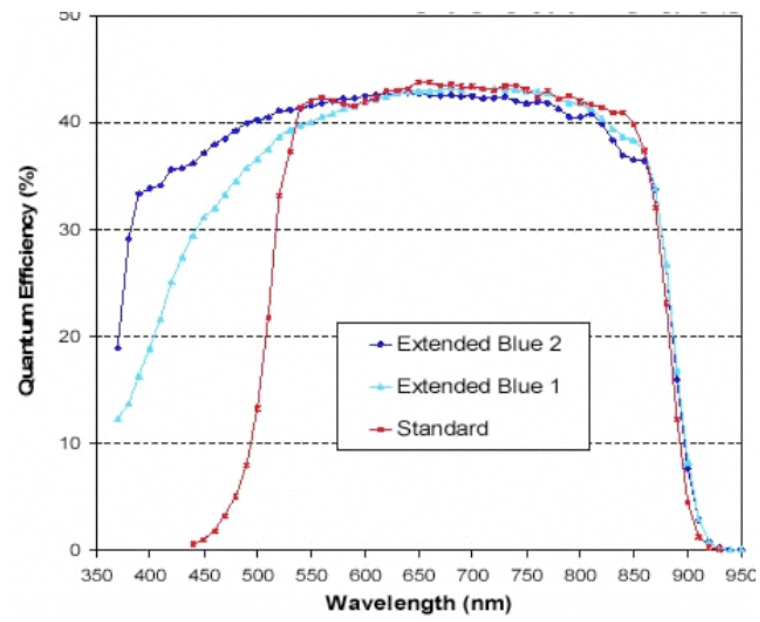

Figure 4. Quantum efficiency of GaAs type photocathodes made by ITT

Most of the readout anodes used in photon counting MCP detectors are serial in nature, i.e. they analyze single events in a short but finite time. Even if this analysis "deadtime" per event could be reduced to $50 \mathrm{~ns}$ with the current generation of fast electronics (analog to digital converters etc.), the best counting rates achieved would be $2 \mathrm{MHz}$ at $10 \%$ deadtime losses. For a future Shack-Hartmann wavefront sensor with 5000 spots of 1000 photons at $1 \mathrm{kHz}$ sampling rate, the total detector event rate would be $5000 \times 1000 \times 1000$ or $5 \mathrm{GHz}$, a factor of 2500 higher rate than the current state of the art in photon counting! To take advantage of the noiseless 
operation of a photon counting detector using MCPs therefore requires a specialized readout that can localize and count the events in a massively parallel way, i.e. pixelated counters. We envisioned fabricating a specialized ASIC mounted behind an MCP that could count and integrate events from the MCP at each pixel and which could be read out digitally and therefore very fast and noiselessly.

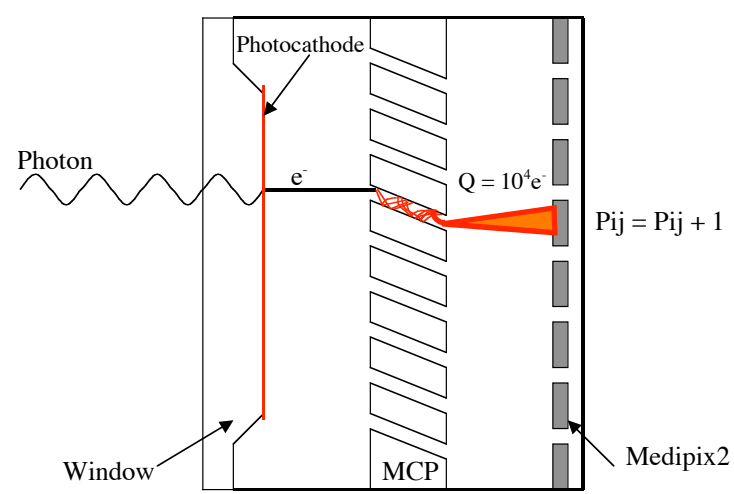

Figure 5. Schematic of a sealed microchannel plate image sensor. The MCP amplifies a single photoelectron and the resultant charge cloud event causes the ASIC pixel counter, Pij, to increment by one.

\section{Medipix2 as a readout anode for MCPs}

Fortunately, we soon discovered that such a device already existed, being designed and constructed by the Microelectronics Group at CERN for the multi-national MEDIPIX collaboration (http://www.cern.ch/medipix).

Our novel detector scheme should achieve the first three of the specific goals listed above with a QE approaching $40 \%$. The detector (Fig. 5) is an MCP image tube with a GaAs photocathode and a new pixelated CMOS readout chip called the "Medipix2" $[4,5]$. Photons interacting with the photocathode release a photoelectron that is proximity focused to the MCP input face. The MCP amplifies this single photoelectron with a gain on the order of $10^{4}$. The resultant charge cloud exits the MCP and lands on the input pad of a Medipix2 pixel where it is counted as one event. The counter will integrate until it is read out in a digital, noiseless process. Also, because the data is digital, it can be read out at $\sim 246 \mathrm{MHz}$ pixel rates, which corresponds to a frame readout time of $266 \mu$ s for the current Medipix 2 chip.

The deadtime per event for the Medipix2 pixel is approximately $500 \mathrm{~ns}$, so each pixel can handle rates up to $200 \mathrm{kHz}$ without too much of a deadtime correction. Over the whole $256 \times 256$ chip, count rates can approach a $13 \mathrm{GHz}$ event rate.

\subsection{First results}

Our first set of Medipix2 chips came mounted on a printed circuit board used for testing by the Medipix collaboration. The board has space for bypass capacitor and resistors and a 68-pin connector. For the eventual vacuum tube application, these parts and connector would be incorporated behind the tube, mounted externally and coupled to the Medipix2 through hermetic feedthroughs. We also designed an MCP detector body that could hold 1 to 3 MCPs at various heights above the Medipix2. The spatial extent of the charge cloud at the Medipix2 input surface is directly proportional to this distance. We could not make this gap smaller than $300 \mu \mathrm{m}$ as the electrical connection to the Medipix chip is through bonding wires on the input surface. There is a voltage bias across this gap to accelerate the electron charge cloud to the Medipix2 to minimize charge spreading. We could also adjust independently the voltage across the $\mathrm{MCP}(\mathrm{s})$ to vary the single event gain (previously calibrated with a simple copper anode). This detector assembly was mounted to a 10-inch vacuum flange mounted on a vacuum chamber and actively pumped down to a pressure of $10^{-6}$ Torr. We used a $\mathrm{Hg}$ pen-ray lamp to illuminate the bare MCP and could adjust the lamp intensity to get $10 \mathrm{cts} . \mathrm{s}^{-1}$ to 500 million cts. $\mathrm{s}^{-1}$.

The Medipix2 chip was controlled and read out using the "MUROS2" control electronics [6] developed for the Medipix consortium by the NIKHEF group and the control software "Medisoft Ver. 4.0" developed by Univ. of Naples Federico II [7]. The only extensive optimization of the Medipix2 settings at Berkeley involved the equalization of the pixel lower charge thresholds per the techniques discussed in reference 5 . 

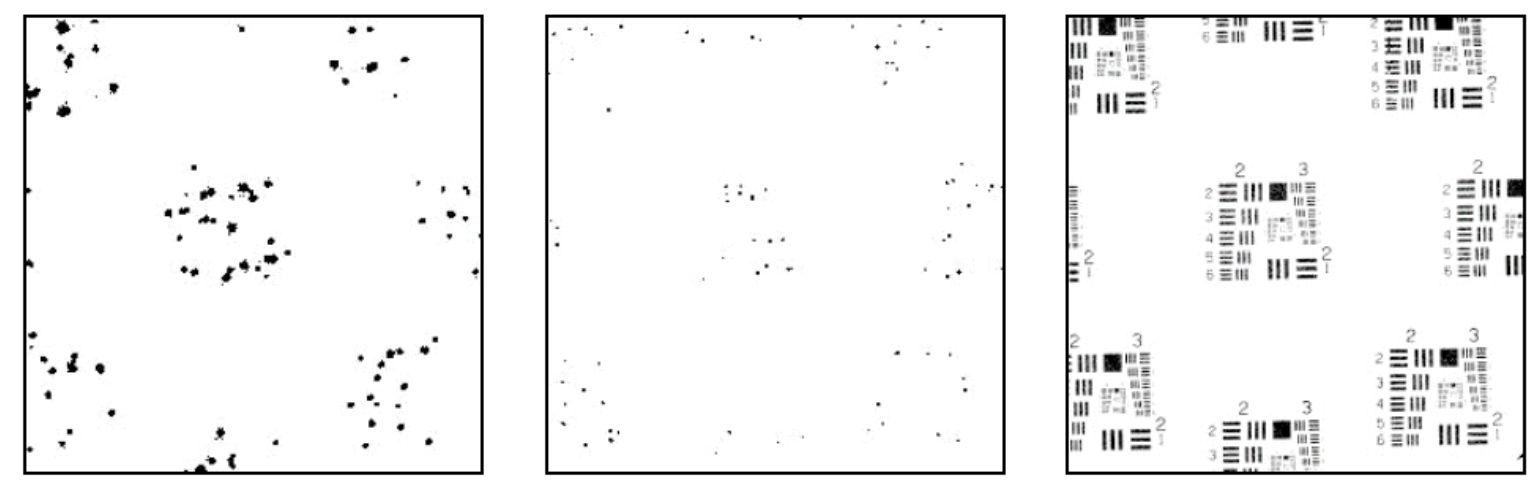

Figure 6. Output images of MCP-Medipix 2 test detector. The two images on the left show single photon events from short $100 \mu \mathrm{s}$ integrations. On the far left, the MCP gain was 200,000 and the rear field voltage was low (300V) resulting in large charge clouds that excited many pixels per photon. In the center image, the MCP gain was reduced and the rear field increased $\left(50 \mathrm{ke}^{-}, 1500 \mathrm{~V}\right)$ resulting in a smaller charge cloud detected on average by only 2.4 pixels. The image on the right is a longer integration (100s) with the bias settings of the center image revealing the Air Force test pattern illuminating the input MCPs.

The initial results (Figs. 6-9) are from a chevron set of two, $33 \mathrm{~mm}$ diameter Photonis MCPs, 10- $\mu \mathrm{m}$ channel diameter pores on $12 \mu \mathrm{m}$ centers, $40: 1 \mathrm{~L} / \mathrm{d}$ thickness, and low resistance (22 $\mathrm{M} \Omega$ per plate). The output end spoiling was 2 channel diameters. For a bias of $1430 \mathrm{~V}$ across the two plates, the average gain was 20,000 , and we tested up to a gain of 200,000 $(1680 \mathrm{~V})$. The rear field between the output of the MCPs and the Medipix 2 could be varied from 0 to $1600 \mathrm{~V}$. At the low gains that we operated the MCP, the lamp brightness for full field illumination did not matter much, as we could use the shutter to take very short exposures of a few microseconds to see single photon events or integrate for many seconds to acquire very deep flat fields.

Figure 6 shows two short integrations and one long integration, which contrast the different data collection modes. The first two are both short enough to see individual photon events coming from the $\mathrm{MCP}$. For the higher gain, lower rear field case (left), the charge clouds are large and extensive enough to trigger many pixels, each recording 1 count (except for event overlaps, where pixels might count 2 events). By lowering the gain and increasing the rear field (middle), the single photon events trigger, on average, 2.4 pixels and have many less overlapping events at this input rate and integration time. Keeping the same settings as the middle, the right image is a long integration where individual events are no longer distinct. This image reveals the Air Force test pattern (AFTP) mask we mounted on the input surface of the MCP. Pattern $3-2$ is just distinguishable (Fig. 9), corresponding to a resolution of $9 \mathrm{lp} / \mathrm{mm}$, exactly the Nyquist limit of a $55 \mu \mathrm{m}$ pixel device.

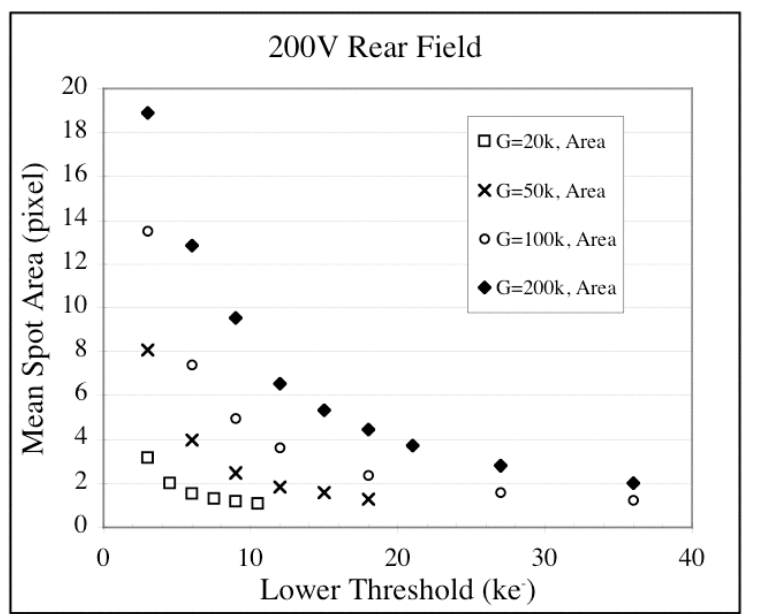

Figure 7. Average event size in pixels vs. lower charge threshold for $4 \mathrm{MCP}$ gains. The rear field is $200 \mathrm{~V}$, leading to rather large event sizes.

Our initial tests probed the extremes of the parameter space for optimizing the detector for $\mathrm{AO}$ performance. Fig. 7 shows the average number of pixels excited per event at a $200 \mathrm{~V}$ rear field at different lower level charge thresholds for various MCP gains. In effect, we are mapping the spatial charge distribution of the average MCP output charge cloud. When normalized by gain, these curves fall 
together indicating that the charge spatial distribution is not a function of gain at these low gains.

We can affect the charge spatial distribution by increasing the rear field. As expected, increasing the field speeds up the transit time of the cloud to the anode and therefore decreases the lateral diffusion. Fig. 8 shows the smaller average event size in pixels for a $1600 \mathrm{~V}$ rear field across the $500 \mu \mathrm{m}$ gap.

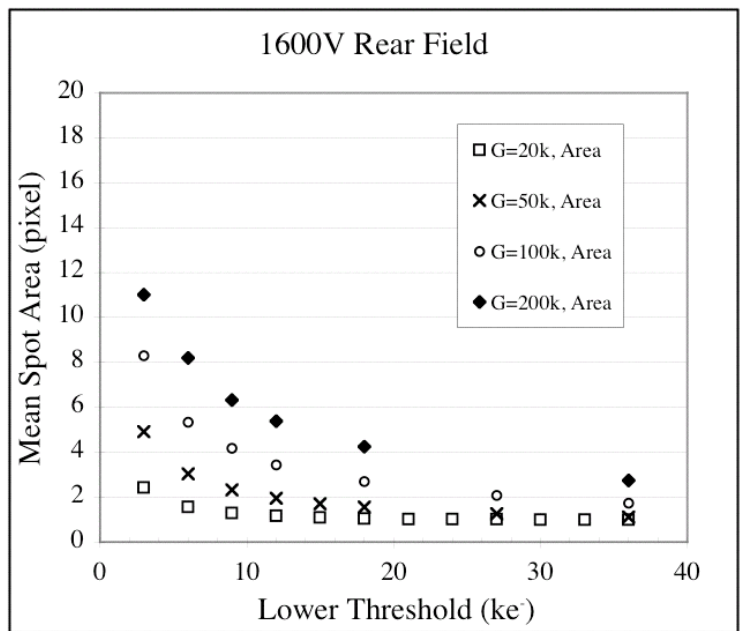

Figure 8. Same as Fig. 7 but with a rear field of 1600 V. The average event size has decreased at all gains.

We have also taken deep flat fields at very high input rates of over 500 million events $\mathrm{s}^{-1}$. The overall uniformity of the flat field is better than $20 \%$ with local variations discernable at the locations of known MCP dead spots and MCP multifiber boundaries, features typical in imaging MCP detector flat fields.

\subsection{Sub-pixel resolution}

If the count rates are low enough such that during a single readout of the Medipix2 detector individual photon events do not overlap, then the possibility exists to use the information gained from the large event sizes to determine the event position centroid to a fraction of a single pixel. This is different than other centroid determinations since it uses a binary centroid calculation: each pixel is either above the lower threshold or not. The right image of Fig. 9 is a 2D histogram of single event centroids determined using this method. The spatial resolution improved by a factor of $\sim 2$ from $9 \mathrm{lp} / \mathrm{mm}$ (left image) to 16 $\mathrm{lp} / \mathrm{mm}$. This method could only be used for count rates less than about 200 events per frame (200$400 \mathrm{kHz}$ ) to avoid overlapping events that result in mis-analyzed events that do not map to the correct position.
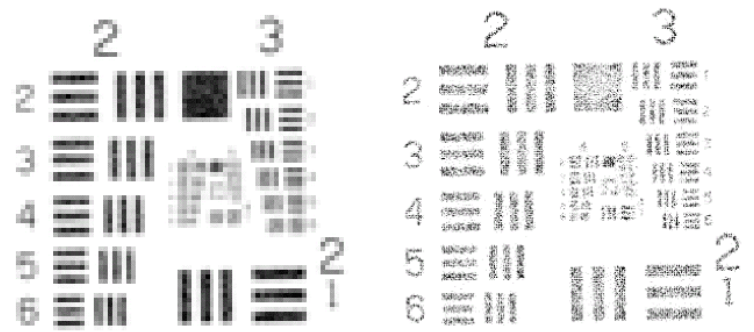

Figure 9. Air Force test pattern mask showing the detector spatial resolution. On the left is the standard Medipix 2 readout where each pixel value is the number of events detected. On the right is a $2 \mathrm{D}$ histogram of detected event centroids using a binary algorithm showing sub-pixel resolution of $16 \mathrm{lp} / \mathrm{mm}$.

\section{Conclusions}

We have described our initial results with an MCP detector read out with a Medipix2 ASIC. The next step for its use as an AO detector is to integrated the individual components into a vacuum tube with a high efficiency optical photocathode such as GaAs. Other applications that can make use of a windowless detector (such as x-ray, far ultraviolet and electron imaging) could adopt this technology immediately by appropriate choice of photocathode.

\section{Acknowledgments}

The authors wish to thank the Medipix Collaboration for the Medipix2 chips, readout hardware and software and for valuable advice. The material presented here is based upon work supported by AURA through the NSF under AURA cooperative agreement \# AST-0132798-SPO\#6(AST-0336888). 


\section{References}

[1] R.K. Tyson, Introduction to Adaptive Optics, SPIE Press, Bellingham, WA, 2000.

[2] R. Angel et al "A Roadmap for the Development of Astronomical Adaptive Optics", July 6, 2000, http://www.noao.edu/dir/ao/

[3] O. H. W Siegmund, Proc. SPIE, 4854, 2003, 190

[4] X. Llopart, M. Campbell, R. Dinapoli, D. SanSegundo, E. Pernigotti, IEEE Trans. Nucl. Sci. NS-49 (2002) 2279

[5] X. Llopart and M. Campbell, Nucl. Instr. and Meth. in Physics Research Section A: Vol. 509, Issues 1-3, 2003,157

[6] D. Bello, M. van Beuzekom, P. Jansweijer, H. Verkooijen and J. Visschers, Nucl. Instru. and Meth. in Physics Research Section A:, Vol 509, 2003, 164

[7] M. Conti, M. Maiorino, G. Mettivier, M. C. Montesi, and P. Russo, IEEE Tran. Nucl. Sci., Vol. 50, No. 4, 2003, 869 